\title{
Role of fenofibrate in restoring angiogenesis in diabetic and control hind limb ischemic rats
}

\author{
Ensieh Salehi ${ }^{1}$, Majid Khazaei ${ }^{1}$ and Bahman Rashidi ${ }^{2}$ \\ ${ }^{1}$ Department of Physiology, Isfahan University of Medical Sciences, Isfahan, Iran \\ ${ }^{2}$ Department of Anatomy, Isfahan University of Medical Sciences, Isfahan, Iran
}

\begin{abstract}
In this study, we examined the effect of fenofibrate, an agonist of peroxisome proliferator-activated receptor $\alpha$ (PPAR $\alpha$ ), on angiogenesis and serum nitric oxide (NO), vascular endothelial growth factor (VEGF) and VEGF receptor-2 (VEGFR-2) in diabetic and control hind limb ischemic rats. Male Wistar rats underwent left hind limb ischemia. The experimental groups consisted of rats: control, diabetic, control receiving fenofibrate $(100 \mathrm{mg} / \mathrm{kg} /$ day, by gavage) and diabetic receiving fenofibrate. After 21 days, blood samples were taken and capillary density and capillary/fiber ratio of the ischemic hind limb muscle was evaluated by immunohistochemistry. Activation of PPARa by fenofibrate restored neovascularization in diabetic and control hind limb ischemic rats $(p<0.05)$. Fenofibrate administration significantly increased serum nitrite concentration, the main metabolite of NO, without significant changes in serum VEGF and VEGFR-2 concentrations. It seems that fenofibrate enhances angiogenesis in hind limb ischemia possibly through increasing of $\mathrm{NO}$ bioavailability and can be considered for treatment of diabetic peripheral vascular diseases in future human studies.
\end{abstract}

Key words: Diabetes - Hind limb ischemia - Fenofibrate - Angiogenesis - Nitric oxide

\section{Introduction}

Peroxisome proliferator-activated receptors (PPARs) are ligand-activated transcription factors introduced in 1990 (Issemann and Green 1990). These nuclear receptors superfamily includes three isotypes of PPARa, PPAR $\gamma$, and $\operatorname{PPAR} \beta / \delta$, which are mapped on different chromosomes (Touyz and Schiffrin 2006; Biscetti et al. 2008). Activation of PPAR $a$ by its ligands may have beneficial effects on lipid metabolism and on reducing the rate of coronary events in diabetic patients (Evans et al. 2000; Playford et al. 2002).

Cardiovascular disease (CVD) is a major cause of morbidity and mortality in diabetic patients (Gaede et al. 2003). Some long-term complications of diabetic vasculopathy are associated with impaired angiogenesis (Waltenberger 2001; Martin et al. 2003). Angiogenesis is a physiological process by which new vessels are formed from preexisting ones. This process is regulated directly and indirectly by several growth factors and cytokines as nitric oxide (NO), vascular

Correspondence to: Majid Khazaei, Department of Physiology, Isfahan University of Medical Sciences, Isfahan, Iran

E-mail: khazaei@med.mui.ac.ir endothelial growth factor (VEGF) and its soluble receptors, VEGF receptor 1 and 2 (VEGFR-1,-2) (Cooke and Losordo 2002; Karamysheva 2008). It is well-documented that PPARs have important role in the process of CVD in diabetic patients (Calkin and Thomas 2008), moreover, they might be involved in regulation of angiogenesis (Biscetti et al. 2009). This study aimed to assess the efficacy of PPAR $a$ activation by fenofibrate in restoring angiogenesis in hind limb ischemia in diabetic and control rats.

\section{Materials and Methods}

\section{Animals}

Male Wistar rats (Pasteur Institute, Iran) with an initial body weight of 180-230 g were used as experiments. They were kept three per cages on a 12-h day/night cycle, with approximate humidity of $60-70 \%$ and room temperature of $20-25^{\circ} \mathrm{C}$. The animals were fed a standard rat chow and had free access to water ad libitum. All experimental procedures were approved by the ethical committee of the Isfahan University of Medical Sciences, Isfahan, Iran. 


\section{Hind limb ischemia model}

The rats were anaesthetized with intraperitoneal injection of ketamine $(75 \mathrm{mg} / \mathrm{kg})$ and xylazine $(7.5 \mathrm{mg} / \mathrm{kg})$. Then, they underwent left hind limb ischemia as previously described (Lloyd et al. 2001; Huang et al. 2008). In brief, the left legs were shaved and locally disinfected. Through a small incision, the left femoral artery was isolated and the proximal and distal portions of left external iliac artery and its side branches were ligated and excised. Subsequently, the skin was closed with 3.0 silk surgical sutures. Then, the animals were returned to their cages.

\section{Experimental protocol}

The animals were randomly assigned into two groups of diabetic and control. Diabetes was induced by intraperitoneal injection of streptozotocin (STZ; Sigma Co. USA) at a dose of $55 \mathrm{mg} / \mathrm{kg}$. STZ was dissolved in a cold $0.1 \mathrm{M}$ citrate buffer ( $\mathrm{pH}$ 4.5). The control group received normal saline injection with the same volume of the medication injected to the case group. After 48 hours, blood glucose levels were measured. Those animals with blood glucose concentrations higher than $16.7 \mathrm{mmol} / \mathrm{l}$ were considered as diabetic (Taniyama et al. 2001). The experimental groups consisted of: Group 1, control rats receiving vehicle; Group 2, control rats receiving daily fenofibrate $(100 \mathrm{mg} / \mathrm{kg} /$ day) by gavage (Katayama et al. 2009); Group 3, diabetic rats receiving vehicle; and Group 4, diabetic rats receiving fenofibrate $(100 \mathrm{mg} / \mathrm{kg} /$ day $)$. The treatments were started one day after the hind limb ischemia operation, and lasted for the next 21 days.

\section{Measurement of capillary density}

At the time of sacrifice, the left gasterocnemious muscles were immediately collected and fixed overnight in $10 \%$ formalin with $\mathrm{pH}=7.2$. The ischemic muscles were embedded in paraffin and cut in $5 \mu \mathrm{m}$ sections. Then, the sections were deparaffinized with xylosine. After hydration in ethanol and washing in deionized water, pretreatment was performed with antigen retrieval in boilling citrate buffer and inhibition of endogenous peroxidase with $3 \% \mathrm{H}_{2} \mathrm{O}_{2}$. Subsequently, for blocking non-specific sites, $50 \mu \mathrm{M}$ of protein block was used for $20 \mathrm{~min}$ at room temperature. After washing in phosphate buffer solution, a rat monoclonal antibody against murine CD31 (Abcam, UK) was used in a 1:50 dilution for antigen detection. At the final stage, capillaries were visualized by 3-3-diaminobezedine (DAB) and were counterstained by using hematoxylin. The capillaries were counted in ten different fields $(\times 400)$ from each tissue preparation and the number of capillaries was counted by two blinded observers. The capillary density was expressed as the number of capillaries per square millimeter. Since muscle atrophy or interstitial edema may overestimate or underestimate capillary density, the capillary/muscle fiber ratio was expressed as well.

\section{Measurement of plasma parameters}

After 12 hours of fasting, blood samples were collected from the retroorbital space. Blood samples were centrifuged with $10,000 \mathrm{rpm}$ for $15 \mathrm{~min}$ to obtain serums. Serum total cholesterol (TC), triglycerides (TG), and high-density lipoprotein cholesterol (HDL-C) were determined using calorimetric assay (Cayman Co., USA). Low-density lipoprotein cholesterol (LDL-C) was calculated by the Friedewald equation. Serum NO concentration was measured using Griess reagent method (Promega Corp., USA) with a limit of detection of $2.5 \mu \mathrm{M}$. Serum VEGF and VEGFR-2 concentrations were measured by Enzyme-linked Immunosorbent assay (ELISA) using available reagents and recombinant standards (R\&D systems, Minneapolis, USA). The minimum sensitivity of VEGF and VEGFR-2 assays are $3.9 \mathrm{pg} / \mathrm{m}$ and $0.027 \mathrm{ng} / \mathrm{ml}$, respectively.

\section{Statistical analysis}

Data are expressed as mean \pm standard error (SE). Comparison of values between groups were evaluated by One-Way analysis of variance (ANOVA) followed by Tukey's post hoc test. Paired data was analyzed by paired $t$-test. $p<0.05$ was considered as statistically significant.

\section{Results}

\section{Plasma parameters}

As shown in Table 1, in diabetic group receiving fenofibrate, serum TG concentration was lower and serum HDL-C was higher than in the non-treated group $(p<0.05)$. Fenofibrate also improved these factors in control group; however, the obtained changes were not statistically significant. Fenofibrate administration did not change blood glucose level in diabetic rats $(26.39 \pm 1.48$ vs. $24.53 \pm 1.15 \mathrm{mmol} / 1 ; p>0.05)$.

\section{Measurement of serum angiogenic factors}

Figure 1 illustrates the concentrations of serum VEGF and VEGFR-2 in all experimental groups. These concentrations were not different among diabetic rats and control groups $(p>$ 0.05). Fenofibrate did not alter serum VEGF and VEGFR-2 values in diabetic and control groups $(p>0.05)$. Diabetic animals had lower serum NO concentration than controls. Fenofibrate could significantly increase serum NO level both in the control and diabetic groups ( $p<0.05$; Figure 2). 
Table 1. Serum lipid profile before and after left hind limb ischemia

\begin{tabular}{lcccccccc}
\hline & \multicolumn{2}{c}{ Total cholesterol $(\mathrm{mg} / \mathrm{dl})$} & \multicolumn{2}{c}{ Triglyceride $(\mathrm{mg} / \mathrm{dl})$} & \multicolumn{2}{c}{ HDL-C $(\mathrm{mg} / \mathrm{dl})$} & \multicolumn{2}{c}{ LDL-C $(\mathrm{mg} / \mathrm{dl})$} \\
\cline { 2 - 9 } & $\mathrm{BI}$ & $\mathrm{AI}$ & $\mathrm{BI}$ & $\mathrm{AI}$ & $\mathrm{BI}$ & $\mathrm{AI}$ & $\mathrm{BI}$ & AI \\
\hline Control & $66.00 \pm 6.79$ & $84.40 \pm 8.74$ & $93.16 \pm 6.72$ & $73.40 \pm 5.88$ & $28.33 \pm 4.19$ & $44.66 \pm 5.01$ & $25.56 \pm 1.94$ & $28.52 \pm 3.66$ \\
Control + Fenofibrate & $61.25 \pm 4.09$ & $80.50 \pm 4.05$ & $81.6 \pm 5.10$ & $75.75 \pm 3.98$ & $29.50 \pm 3.30$ & $34.50 \pm 2.71$ & $20.65 \pm 1.71$ & $27.23 \pm 3.12$ \\
\cline { 2 - 9 } Diabetic & $70.33 \pm 5.44$ & $72.25 \pm 7.06$ & $81.80 \pm 15.02$ & $71.25 \pm 12.37$ & $26.00 \pm 3.55$ & $33.40 \pm 2.76$ & $19.63 \pm 2.44$ & $20.76 \pm 2.56$ \\
Diabetic + Fenofibrate & $69.18 \pm 4.46$ & $89.66 \pm 7.82$ & $93.45 \pm 7.74$ & $66.33 \pm 3.56^{*}$ & $28.60 \pm 2.75$ & $42.83 \pm 4.51^{*}$ & $22.49 \pm 1.94$ & $28.25 \pm 1.89$ \\
\hline
\end{tabular}

Data are expressed as mean $\pm \mathrm{SE}$; ${ }^{\star} p<0.05$ compared to BI. AI, after left hind limb ischemia; BI, before ischemia; HDL-C, high-density lipoprotein cholesterol; LDL-C, low-density lipoprotein cholesterol.

Angiogenesis assay in ischemic hind limb by immunohistochemistry

Angiogenesis was evaluated by the capillary density and capillary/fiber ratio of the ischemic hind limb muscle harvested three weeks after treatment. Our results revealed

\section{$\mathbf{A}$}

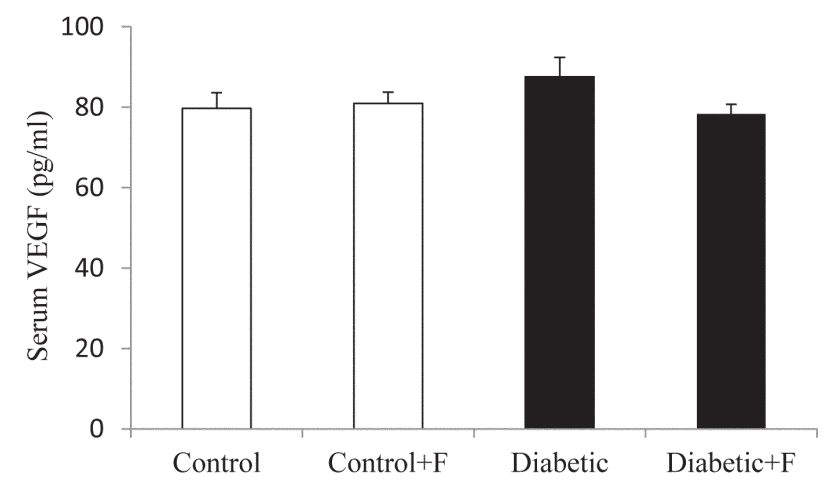

B

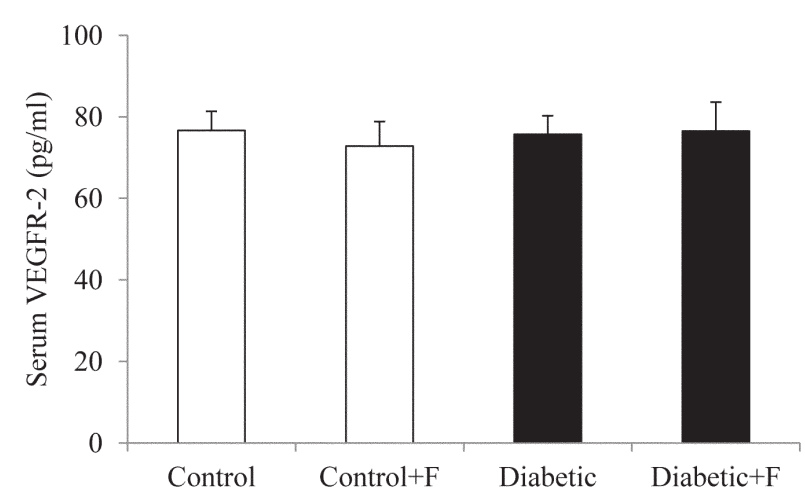

Figure 1. Effect of fenofibrate on serum VEGF (A) and VEGFR-2 (B) concentrations. Fenofibrate could not change serum VEGF and VEGFR-2 concentrations. Values are expressed as mean $\pm \mathrm{SE}$, $n=6$ per group. F, fenofibrate; VEGF, vascular endothelial growth factor; VEGFR-2, VEGF receptor 2. that the capillary density (Figure 3A) and capillary/fiber ratio (Figure $3 \mathrm{~B}$ ) in hind limb ischemia were significantly reduced in diabetic animals compared to the control group $(p<0.05)$. Samples of immunohistochemical staining by anti-CD31 antibody in ischemic legs are presented in Figure 4. As shown in Figures 3 and 4, fenofibrate significantly restored neovascularization in the ischemic leg of control and diabetic rats.

\section{Discussion}

In this study, we demonstrated that the neovascularization in ischemic leg of diabetic animals was lower than controls, and treatment of control and diabetic rats by fenofibrate, as a synthetic agonist of PPAR $\alpha$, could lead to neovascularization in ischemic leg. Furthermore, fenofibrate increased serum NO concentration without changes in serum VEGF and VEGFR-2 concentrations.

Beneficial effects of fenofibrate on lipid metabolism are documented in several studies among hyperlipidemic sub-

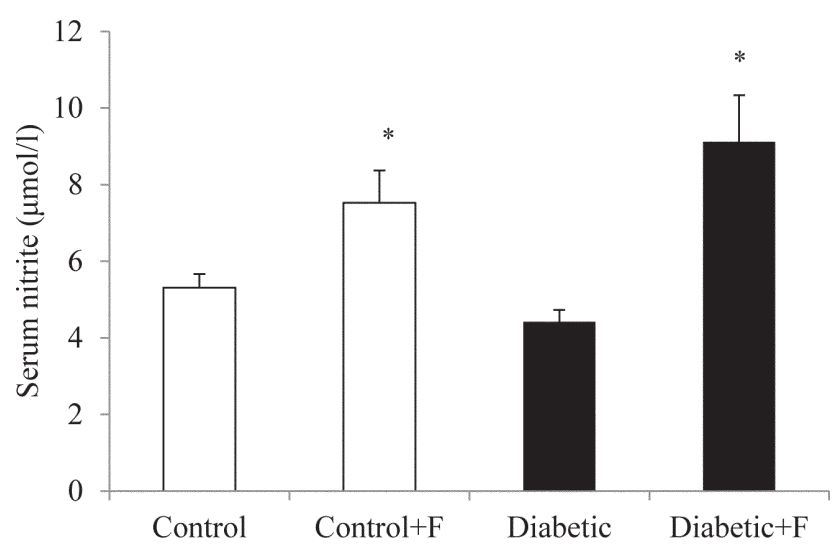

Figure 2. Effect of fenofibrate on NO serum concentration ( $\mu \mathrm{mol} / \mathrm{l})$ in control and diabetic animals. Fenofibrate improved serum nitrite concentration in both groups. Values are expressed as mean $\pm \mathrm{SE}$, $n=6$ per group; ${ }^{\star} p<0.05$ compared to non-treated group. F, fenofibrate. 
A

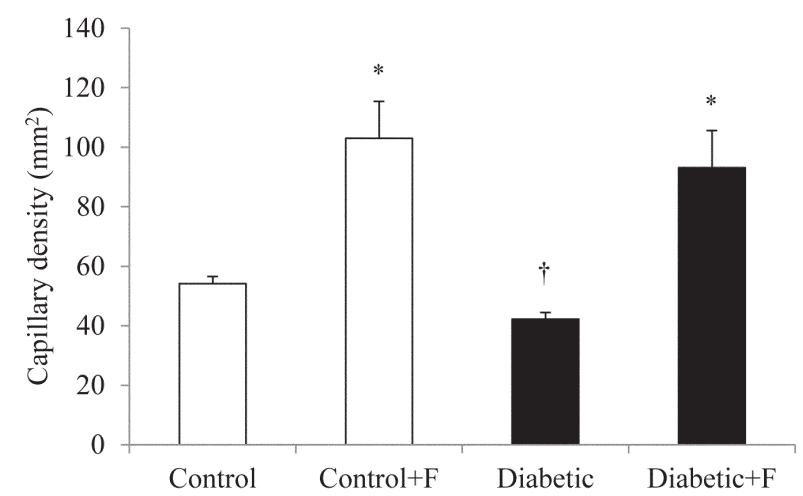

B

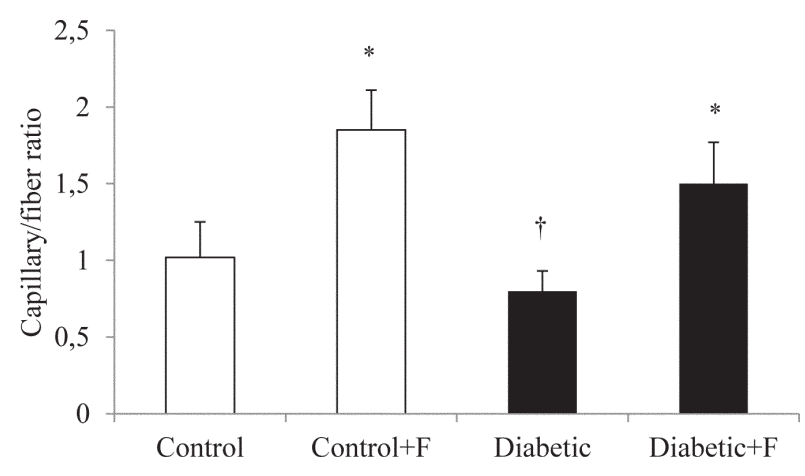

Figure 3. Capillary density (A) and capillary/fiber ratio (B) in hind limb ischemia of control and diabetic animals and effect of fenofibrate (F). Values are expressed as mean $\pm \mathrm{SE}, n=6$ per group; ${ }^{*} p<0.05$ compared to the untreated group; $\dagger p<0.05$ compared to the control group.
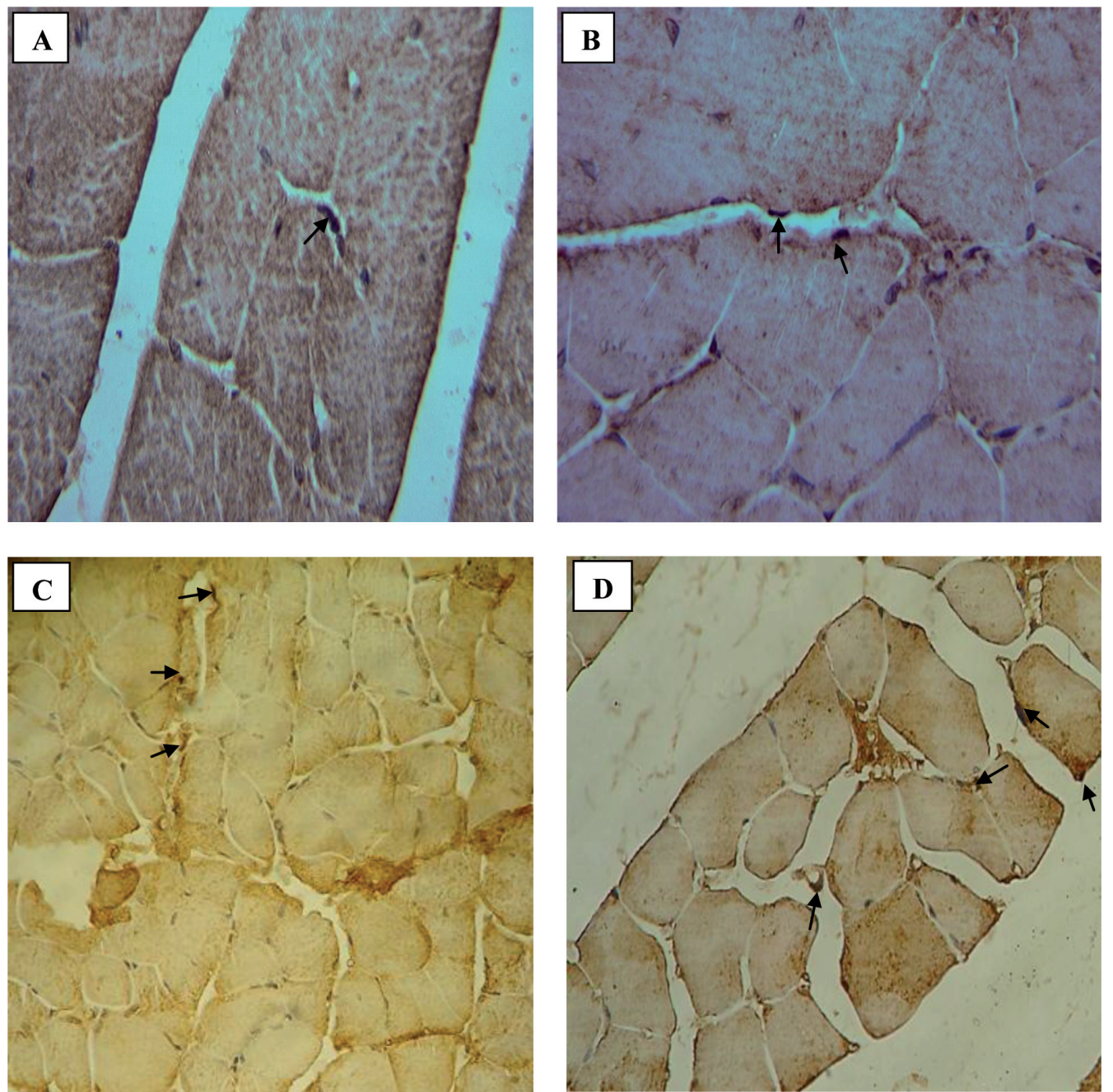

Figure 4. Representative photographs of immunohistochemical staining $(\times 400)$ with anti-CD31 monoclonal antibody in diabetic (A), control (B), diabetic received fenofibrate (C), and control received fenofibrate (D) group. Arrows indicate CD31-positive cells. Fenofibrate restored angiogenesis in control and diabetic groups. 
jects (Evans et al. 2000; Forcheron et al. 2002; Playford et al. 2002). In the present study, the animals received normal diet, not high-fat diet, however, we found that fenofibrate improved serum HDL-C and decreased TG concentrations in diabetic animals. This finding is consistent with some previous studies. The possible mechanisms attributing the beneficial effects of PPAR $\alpha$ agonists on lipid profile include: decreasing of hormone-sensitive lipase activity, promoting uptake and oxidation of fatty acid in muscles, lowering hepatic de novo lipogenesis and modification of the expression of various key genes involved in the metabolisms of liver fatty acids (Guo et al. 2001; Forcheron et al. 2002).

In recent years, studies have been performed on the angiogenic effects of PPARs (Biscetti et al. 2009). Angiogenesis is a physiological response to hypoxia and defined as formation of new blood vessels from pre-existing ones (Waltenberger 2001). PPARa agonists, especially fenofibrate, inhibit pathological angiogenesis associated with tumor growth in mice (Panigrahy et al. 2008). It also suppresses cell proliferation and migration stimulated by angiogenic factors in a dose-dependent manner (Varet et al. 2003). In contrast, in the present study, we found that fenofibrate administration could restore ischemiainduced angiogenesis in skeletal muscle of diabetic and control rats. Likewise, fenofibrate treatment significantly increased serum NO concentration, which is a known angiogenic factor (Cooke and Losordo 2002). Our results were consistent with the previous studies. A recent study on normal C57BL/6J mice revealed that fenofibrate promotes angiogenesis in ischemic limb of normal mice by activation of NO pathway in endothelial cells (Katayama et al. 2009). They indicated that observed angiogenic response is independent of changing in serum triglyceride levels. Another study in corneal model of angiogenesis demonstrated that activation of PPARa enhanced neovascularization indirectly via upregulation of the angiogenic factors (Biscetti et al. 2008). Interestingly, inhibition of VEGF activity did not suppressed induced neovascularization by PPARa. Thus, they suggested that other angiogenic factors might be involved in PPARa-induced angiogenesis. In the present study, we found higher level of serum NO concentration in bezafibrate-treated groups both in control and diabetic animals. Proangiogenic effect of NO is already indicated (Namba et al. 2003; Luque et al. 2006). It also mediates the angiogenic response to VEGF or other angiogenic factors (Fukumura et al. 2001). Thus, our findings suggest that the angiogenic effect of fenofibrate is possibly derived via NOdependent pathway, although more studies are necessary for clarification.

In conclusion, fenofibrate restored neovascularization in hind limb ischemic diabetic and control rats. Therefore, it can be considered for prevention and/or treatment of peripheral vascular complications in diabetic patients. Increase in the
NO bioavailability is probably responsible for this beneficial effect; however, more studies are needed to confirm these findings.

Acknowledgements. This study was supported by grant (No. 188138) from the Isfahan University of Medical Sciences.

\section{References}

Biscetti F., Gaetani E., Flex A., Aprahamian T., Hopkins T., Straface G., Pecorini G., Stigliano E., Smith R. C., Angelini F., Castellot J. J., Jr., Pola R. (2008): Selective activation of peroxisome proliferator-activated receptor (PPAR)alpha and PPAR gamma induces neoangiogenesis through a vascular endothelial growth factor-dependent mechanism. Diabetes 57, 1394-1404 http://dx.doi.org/10.2337/db07-0765

Biscetti F., Straface G., Pitocco D., Zaccardi F., Ghirlanda G., Flex A. (2009): Peroxisome proliferator-activated receptors and angiogenesis. Nutr. Metab Cardiovasc. Dis. 19, 751-759 http://dx.doi.org/10.1016/j.numecd.2009.04.011

Calkin A. C., Thomas M. C. (2008): PPAR agonists and cardiovascular disease in diabetes. PPAR Res. 2008, 245410 http://dx.doi.org/10.1155/2008/245410

Cooke J. P., Losordo D. W. (2002): Nitric oxide and angiogenesis. Circulation 105, 2133-2135 http://dx.doi.org/10.1161/01.CIR.0000014928.45119.73

Evans M., Anderson R. A., Graham J., Ellis G. R., Morris K., Davies S., Jackson S. K., Lewis M. J., Frenneaux M. P., Rees A. (2000): Ciprofibrate therapy improves endothelial function and reduces postprandial lipemia and oxidative stress in type 2 diabetes mellitus. Circulation 101, 1773-1779 http://dx.doi.org/10.1161/01.CIR.101.15.1773

Forcheron F., Cachefo A., Thevenon S., Pinteur C., Beylot M. (2002): Mechanisms of the triglyceride- and cholesterol-lowering effect of fenofibrate in hyperlipidemic type 2 diabetic patients. Diabetes 51, 3486-3491 http://dx.doi.org/10.2337/diabetes.51.12.3486

Fukumura D., Gohongi T., Kadambi A., Izumi Y., Ang J., Yun C. O., Buerk D. G., Huang P. L., Jain R. K. (2001): Predominant role of endothelial nitric oxide synthase in vascular endothelial growth factor-induced angiogenesis and vascular permeability. Proc. Natl. Acad. Sci. U.S.A. 98, 2604-2609 http://dx.doi.org/10.1073/pnas.041359198

Gaede P., Vedel P., Larsen N., Jensen G. V., Parving H. H., Pedersen O. (2003): Multifactorial intervention and cardiovascular disease in patients with type 2 diabetes. N. Engl. J. Med. 348, 383-393 http://dx.doi.org/10.1056/NEJMoa021778

Guo Q., Wang P. R., Milot D. P., Ippolito M. C., Hernandez M., Burton C. A., Wright S. D., Chao Y. (2001): Regulation of lipid metabolism and gene expression by fenofibrate in hamsters. Biochim. Biophys. Acta 1533, 220-232

Huang P. H., Sata M., Nishimatsu H., Sumi M., Hirata Y., Nagai R. (2008): Pioglitazone ameliorates endothelial dysfunction and restores ischemia-induced angiogenesis in diabetic mice. Biomed. Pharmacother. 62, 46-52 http://dx.doi.org/10.1016/j.biopha.2007.06.014 
Issemann I., Green S. (1990): Activation of a member of the steroid hormone receptor superfamily by peroxisome proliferators. Nature 347, 645-650 http://dx.doi.org/10.1038/347645a0

Karamysheva A. F. (2008): Mechanisms of angiogenesis. Biochemistry (Mosc.) 73, 751-762 http://dx.doi.org/10.1134/S0006297908070031

Katayama A., Yamamoto Y., Tanaka K., Matsubara K., Sugitani M., Fujihara S., Harada S., Kaetsu Y., Yoshida A., Hisatome I. (2009): Fenofibrate enhances neovascularization in a murine ischemic hind limb model. J. Cardiovasc. Pharmacol. 54, 399-404 http://dx.doi.org/10.1097/FJC.0b013e3181bad05d

Lloyd P. G., Yang H. T., Terjung R. L. (2001): Arteriogenesis and angiogenesis in rat ischemic hind limb: role of nitric oxide. Am. J. Physiol. Heart Circ. Physiol. 281, H2528-2538

Luque C. D., Vargas R. H., Romo E., Rios A., Escalante B. (2006): The role of nitric oxide in the post-ischemic revascularization process. Pharmacol. Ther. 112, 553-563 http://dx.doi.org/10.1016/j.pharmthera.2006.05.003

Martin A., Komada M. R., Sane D. C. (2003): Abnormal angiogenesis in diabetes mellitus. Med. Res. Rev. 23, 117-145 http://dx.doi.org/10.1002/med.10024

Namba T., Koike H., Murakami K., Aoki M., Makino H., Hashiya N., Ogihara T., Kaneda Y., Kohno M., Morishita R. (2003): Angiogenesis induced by endothelial nitric oxide synthase gene through vascular endothelial growth factor expression in a rat hind limb ischemia model. Circulation 108, 2250-2257 http://dx.doi.org/10.1161/01.CIR.0000093190.53478.78

Panigrahy D., Kaipainen A., Huang S., Butterfield C. E., Barnes C. M., Fannon M., Laforme A. M., Chaponis D. M., Folkman J.,
Kieran M. W. (2008): PPARalpha agonist fenofibrate suppresses tumor growth through direct and indirect angiogenesis inhibition. Proc. Natl. Acad. Sci. U.S.A. 105, 985-990 http://dx.doi.org/10.1073/pnas.0711281105

Playford D. A., Watts G. F., Best J. D., Burke V. (2002): Effect of fenofibrate on brachial artery flow-mediated dilatation in type 2 diabetes mellitus. Am. J. Cardiol. 90, 1254-1257 http://dx.doi.org/10.1016/S0002-9149(02)02847-3

Taniyama Y., Morishita R., Aoki M., Nakagami H., Yamamoto K., Yamazaki K., Matsumoto K., Nakamura T., Kaneda Y., Ogihara T. (2001): Therapeutic angiogenesis induced by human hepatocyte growth factor gene in rat and rabbit hind limb ischemia models: preclinical study for treatment of peripheral arterial disease. Gene Ther. 8, 181-189 http://dx.doi.org/10.1038/sj.gt.3301379

Touyz R. M., Schiffrin E. L. (2006): Peroxisome proliferator-activated receptors in vascular biology-molecular mechanisms and clinical implications. Vascul. Pharmacol. 45, 19-28 http://dx.doi.org/10.1016/j.vph.2005.11.014

Varet J., Vincent L., Mirshahi P., Pille J. V., Legrand E., Opolon P., Mishal Z., Soria J., Li H., Soria C. (2003): Fenofibrate inhibits angiogenesis in vitro and in vivo. Cell. Mol. Life Sci. 60, $810-819$ http://dx.doi.org/10.1007/s00018-003-2322-6

Waltenberger J. (2001): Impaired collateral vessel development in diabetes: potential cellular mechanisms and therapeutic implications. Cardiovasc. Res. 49, 554-560 http://dx.doi.org/10.1016/S0008-6363(00)00228-5

Received: September 15, 2011

Final version accepted: January 9, 2012 